\title{
Color moment and gray level co-occurrence matrix in classification of soil organic matter for patchouli plantation
}

\author{
Candra Dewi ${ }^{1}$, Mudawikawi Akbar Grahadhuita ${ }^{2}$, Lailil Muflikhah ${ }^{3}$ \\ 1,2,3 Informatics Department, University of Brawijaya, Indonesia \\ ${ }^{1}$ Essential Oil Institute, University of Brawijaya, Indonesia
}

\begin{tabular}{l}
\hline Article Info \\
\hline Article history: \\
Received Dec 26, 2019 \\
Revised Feb 27, 2020 \\
Accepted Mar 12, 2020 \\
\hline
\end{tabular}

Keywords:

Color moment

GLCM

MKNN

Patchouli

Soil organic matter

\begin{abstract}
Patchouli is one of the essential plants that have the most potential and widely cultivated in Indonesia. Patchouli is greedily absorbing soil nutrients and organic matter. Therefore, the selection of soil with high organic matter will maximize the patchouli's productivity. This paper aims to facilitate soil's organic matter identification by classifying soil image based on the combination of color and texture features. The color feature extraction was done using the Color Moments method and the texture feature was done using Gray Level Co-occurrence Matrix (GLCM) method. The selection of features was performed to obtain the best combination of color and texture features. The selected features then was used as input of classification by using Modified K-Nearest Neighbor (MKNN). The samples of soil that used as data were taken from several districts in Blitar, East Java province. The testing result of this research showed the highest accuracy of 93,33\% by using 180 training data, and also particular color and texture feature combination.
\end{abstract}

Copyright $(0) 2020$ Institute of Advanced Engineering and Science. All rights reserved.

\section{Corresponding Author:}

Candra Dewi,

Informatics Department,

University of Brawijaya, Indonesia.

Email: dewi_candra@ub.ac.id

\section{INTRODUCTION}

Patchouli (Pogostemon cablin) is one of the most essential plants that are cultivated because it produces natural patchouli oil which is needed in the cosmetics, perfume and antiseptic industries. Patchouli is known as a greedy plant in the absorption of organic matter and nutrients, especially elements of nitrogen $(\mathrm{N})$, phosphorus (F), and potassium (K). Therefore, to obtain good quality of patchouli plants, it must be ensured that the soil used for planting has sufficient organic matter and nutrients [1]. Thus, identification from the beginning of the content of soil organic matter needs to be carried out before planting. In contrast to the methods commonly used for checking organic matter through soil laboratory test, this research offered a new method for identification by utilizing soil images. Specifically, the purpose of this study was 1) to obtain the best combination of soil color and texture feature extracted from soil image 2) to calculate the accuracy of the classifiying of organic matter content in soil through soil image.

Research to detect nutrients using soil imagery has been carried out by [2-3]. However, in the study by [2] the soil chromatogram image was used and only resulted the new features were extracted from the image. Whereas in research [3] used imaging spectroscopy and produced characterization of spatial variability of soil organic carbon and nitrogen at soil aggregate scale. The other research related to the classification and mapping of soil organic carbon has also been carried out using satellite images and airborne image [4-9]. Although this technique is quite effective, but not all satellite imagery data is easily obtained especially for certain areas with a narrow area coverage. In addition to satellite data with high resolution is often quite expensive. While aerial photo data also requires a lot of time and cost. 
In contrast to the chromatogram image, spectroscopy image, and satellite image, the use of other images such as images taken using a camera needs to be further investigated for their efficiency and accuracy. Therefore, this study examines the effectiveness of images taken by using a DLSR camera. Research conducted by [10-11] shows that the use of image analysis results in a high correlation and quite good accuracy. This shows the effectiveness of using images in predicting organic soil carbon content.

Based on [12], soil color shows the indicator of quantity of organic matter, drainage and aeration. Soils containing high organic materials have different colors and texture, therefore the soil image taken also has a variety of pixel values. It's also has been explained that soils with high organic matter have different colors although the image has a nearly uniform pixel value. Usually, the soil image taken has almost the same pixel value variation. It means that the similar pixel value allows data to be recognized as the same class when it should be in a different class. Therefore, calculating the distribution of values from a set of pixels is a way that can be used to get the appropriate feature. For this reason, a technique is needed to extract the right features and recognition techniques in the identification process. This study uses the color moment method that utilizes information on low-order moments. Color moments encode both shape and color information so that it is a feature that is suitable for images with changing lighting conditions. Several studies conducted by [13-17] showed that the use of color moment can improve recognition accuracy if there is a change in viewpoint and illumination in the image.

Although the color moment can work well on changing lighting conditions, but it is unable to overcome occlusion. Color moment also cannot recognize differences texture in the image, even though the soil with different organic ingredients will have different textures. Therefore, in this study we applied a combination of color moment features and texture features. The texture feature used in the study was extracted by the gray level co-occurrence matrix (GLCM) method to obtain the level of contrast, granularity and exposure of an area in the image. This feature has been widely used in research with good results [18-21]. In addition, the advantages of GLCM are considering the relevance between pixels in various orientation directions so that more detailed and complete fiter information will be obtained. Beside, we also perform the experiment to obtain the optimum combination of color moment and GLCM features.

Furthermore, we apply Modified K-Nearest Neighbor (MKNN) method to qualify the organic matter into three classes, namely very low, low, and moderate. K-Nearest Neighbor (KNN) uses the distance function to get the shortest distance from neighboring pixels in getting the selected class [22-23]. Modified KNN is the improvement of the KNN method by adding the weight for the closest neighbord. MKNN proved to be successful in various studies [24-27]. The improved that we used is by adding validity calculation for training data according to the $\mathrm{K}$ value and calculation of weight voting for each closest neighbor as much as $\mathrm{K}$.

\section{RESEARCH METHOD}

\subsection{Determining image feature of soil}

Patchouli will grow well on soil with the type regosol, red latosol or aluviall. The soil structure must be loose and deep, fertile and contains a rich of organic matter. The soil texture is sandy clay or dusty clay [28]. The three properties of soil as indicator of the content of organic matter, namely, soil color, soil texture and soil structure [29]. Soil that has black or dark color indicates a high content of organic matter. Soil texture shows a relative comparison of sand, dust and clay fractions, each of which has a different size. The United States Department of Agriculture (USDA) divides the soil texture into 3 general textures, namely sandy land, clay soil and muddy soil. Soil structure show the arrangement of primary soil particles (sand, dust, and clay) to aggregates. These two physical properties mainly use soil particles from large (rough) to small (fine) sizes.

As mentioned that soil color and soil texture can be used as the indicator of soil organic content. The properties of soil can be extracted from the soil image by using specified method. This research use color moment method for color feature extraction, and Grey Level Co-occurrence Matrix (GLCM) for texture feature extraction. The classification process needs class reference as validation value of organic matter class. There are five criteria or category of soil organic matter has been classified by Center of Soil and Agroclimate Research, Bogor, Indonesia and can be seen in Table 1. We used these criteria as class reference in classification process using MKNN.

Table 1. Criteria for soil organic matter

\begin{tabular}{cc}
\hline Organic Matter $(\%)$ & Criteria \\
\hline$<1,00$ & Very low \\
$1,00-2,00$ & Low \\
$2,10-4,20$ & Moderate \\
$4,30-6,00$ & High \\
$>6,00$ & Very high \\
\hline
\end{tabular}

Indonesian J Elec Eng \& Comp Sci, Vol. 19, No. 2, August 2020 : 983 - 991 


\subsection{Data preparation}

The image of the soil was taken from the area where essential was planted and other location that were not planted with essential plant. The location of the soil sampling data is in central part of Blitar district, which is in the Kesamben sub-district, Wlingi sub-district, Kanigoro sub-district, Selopuro sub-district, and Selorejo sub-district. The soil sample taken is soil at a depth of $30 \mathrm{~cm}$ from the surface. The soil is then dried by placing them in the open space in a sun proof room for two days. This is done to dry soil samples without damaging the organic matter content in the sample. The dry soil sample is then compacted and taken the image of using an DLSR camera in handmade studio with a 25-watt lighting in the rear position of the audio studio. The image is then cropped with a size of $300 \times 300$ pixels to be used as training and testing data. The example of soil image is shown in Figure 1.

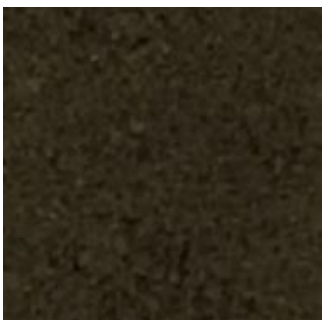

(a)

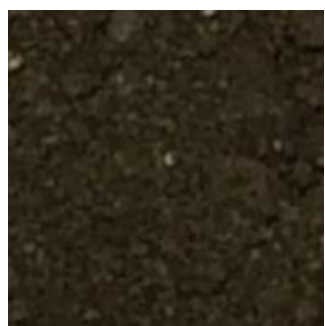

(b)

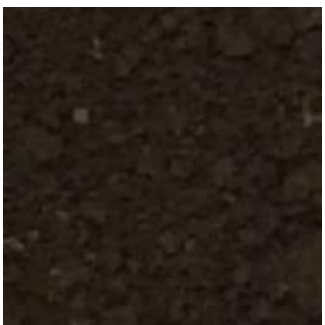

(c)

Figure 1. Example of soil image with criteria (a) very low (b) low (c) moderate

The soils were also tested in the laboratory to obtain the data of organic matter contained in the soil. Tests were carried out in the Soil Chemistry Laboratory of the Faculty of Agriculture, University of Brawijaya. This laboratory test is used as validation data in the testing phase of proposed model. The classes starting from very low, low and moderate. The example of laboratory test from soil samples are presented in Table 2 .

Table 2. Laboratory test of soil sample

\begin{tabular}{ccc}
\hline Soil Sample & Organic Matter $(\%)$ & Class \\
\hline Kanigoro 1 & 1,27 & Low \\
Kanigoro 2 & 0,99 & Very Low \\
Kanigoro 3 & 1,86 & Low \\
Kesamben & 2,75 & Moderate \\
Selopuro 2 & 0,84 & Very Low \\
Selopuro 3 & 0,31 & Very Low \\
Selorejo 1 & 2 & Low \\
Selorejo 2 & 1,61 & Low \\
Selorejo 3 & 1,04 & Low \\
Selorejo 4 & 1,44 & Low \\
Soil 1 & 2,18 & Moderate \\
Soil 5 & 2,44 & Moderate \\
Soil 7 & 1,74 & Low \\
Soil 8 & 1,73 & Low \\
Soil 10 & 1,86 & Low \\
Soil 12 & 3,57 & Moderate \\
Soil 14 & 3,67 & Moderate \\
Soil 17 & 2,32 & Moderate \\
Soil 18 & 1,97 & Moderate \\
Soil 20 & 0,86 & Very Low \\
\hline
\end{tabular}

\subsection{Research procedure}

General procedure of the identification process of organic matter is shown at Figure 2. The input is soil image and the output is class of organic matter. The process consists of several stages, namely feature extraction stage which consists of extracting color features using the color moment method and texture feature extraction using the gray level co-occurrence matrix (GLCM) method, initialization of K value, calculation of the validity of training data, and the classification process of testing data using the MKNN method. 


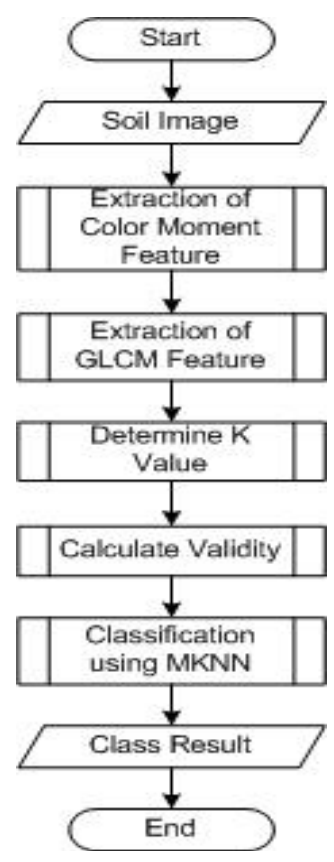

Figure 1. Flow chart of the proposed method

Color Moments extract color features of an image without being influenced by differences in lighting and image size. This study uses two moments, namely the first moment $(\mu)$ which represents the average color (mean) and the second moment $(\sigma)$ which is the standard deviation (STD) [30]. This moments can be calculated by using (1) for mean value and (2) for deviation standard.

$$
\begin{aligned}
& \mu_{c}=\frac{1}{M N} \sum_{i=1}^{M} \sum_{j=1}^{N} P_{i, j}^{c} \\
& \sigma_{c}=\left[\frac{1}{M N} \sum_{i=1}^{M} \sum_{j=1}^{N}\left(P_{i, j}^{c}-\mu_{c}\right)^{2}\right]^{1 / 2}
\end{aligned}
$$

Where $\mu_{c}$ is moment, $\sigma_{c}$ is deviation standard, $c$ is color componen, $P_{i, j}^{c}$ is pixel value $(\mathrm{i}, \mathrm{j})$ on the color componen, $M$ is the height of image and $N$ is the width of image.

Coocurence calculate the number of occurrences on one level of intensity values of neighboring pixels with one level of intensity of another pixel in a certain distance and angle orientation $(\theta)$. Distance is expressed in pixels and orientation is expressed in degrees. The orientation is formed in four angles with $45^{\circ}$ interval of angles, namely $0^{\circ}, 45^{\circ}, 90^{\circ}$, and $135^{\circ}$. This study uses four orientation in calculation of GLCM features and the distance between pixels is set at one pixel. The coocurrence is displayed at Error! Reference source not found.

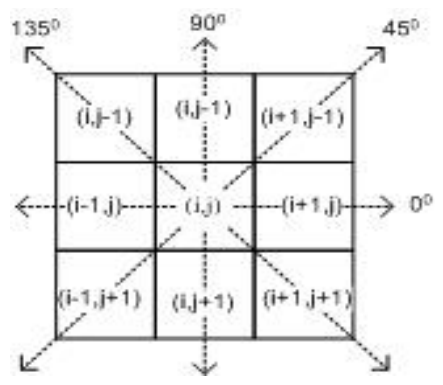

Figure 2. Neighboring relations between pixels 
Gray Level Co-Occurence Matrix (GLCM) is a second-order statistical texture feature extraction method. Texture features extracted for this research are Angular Second Moment (ASM), Contrast, Correlation, Inverse Difference Moment (IDM), and Entropy features. ASM is used to measure of the uniformity of texture in an image. The ASM value itself is high at the time of distribution of the gray degree of a constant image or periodically. This feature can be calculated using (3).

$$
A S M=\sum_{i, j=0}^{N-1}\{p(i, j)\}^{2}
$$

Contrast show a contrast measure of an image or the number of local variations found in that image. Contrast is the difference of moments of the GLCM matrix, which is the difference between the smallest and largest values of adjacent pixels. Contrass value can be calculated by using (4).

$$
\text { Contrast }=\sum_{i, j=0}^{N-1} p(i-j)^{2}
$$

The number of Correlation is a measure of the linear dependence of the gray level of an image on a pixel neighbor and can be calculated by using (5).

$$
\text { Correlation }=\frac{\sum_{i, j=1}^{N}(i, j)\left(p(i, j)-\mu_{i} \mu_{j}\right)}{\sigma_{i} \sigma_{j}}
$$

IDM is a measure of the homogeneity of an image. The IDM value is inversely proportional to the contrast value and can be calculated using (6).

$$
I D M=\sum_{i, j=0}^{N-1} \frac{p(i, j)}{1+(i-j)^{2}}
$$

Entropy is a measure of non-rule in an image. Entropy has a high value if the image does not have uniform texture and many elements of the GLCM Matrix have a low value. Complex textures tend to have high entropy values. Entropy is inversely proportional to ASM. This moment can be calculated by using (7).

$$
\text { Entropy }=-\sum_{i, j=0}^{N-1} p(i, j) \log p(i, j)
$$

Where $p(i, j)$ is GLCM value on element $(i, j), N$ is a number of gray levels used in quantization process, $\mu$ is GLCM mean, $\sigma^{2}$ is the variance of the intensities of all reference pixels in the relationship that contributed to the GLCM. After we got the features, the next process was calculation of the validity of training data. This process is based on neighbor values from training data and can be done using (8).

$$
\operatorname{validity}(x)=\frac{1}{H} \sum_{i=1}^{H} S\left(\operatorname{lbl}(x), \operatorname{lbl}\left(N_{i}(x)\right)\right.
$$

Where $H$ is the number of closest point, $\operatorname{lbl}(\mathrm{x})$ is class of $\mathrm{x}, \mathrm{N}_{\mathrm{i}}(\mathrm{x})$ is class label of the nearest point from $\mathrm{x}$. The $S$ function is used to calculate the similarity between the point $\mathrm{x}$ and the-i neighbor. The value of the $\mathrm{S}$ function is shown in (9).

$$
S(a, b)= \begin{cases}1 & a=b \\ 0 & a \neq b\end{cases}
$$

Where $\boldsymbol{a}$ is class of training data, $\boldsymbol{b}$ is neighboring class of training data.

The process then is continued with classification using Modified K-Nearest Neighbor (MKNN). MKNN is the improvement of the K-Nearest Neighbor (KNN) method by adding a validity calculation to match the $\mathrm{K}$ value. Then weight voting is calculated for each nearest neighbor as much as $\mathrm{K}$ with the calculated validity value of training data [31]. The algorithm of MKNN is as follows:

a) Calculate the distance of new (predicted) data with all existing data

b) Sort the data based on the distance and find the $\mathrm{k}$ data that has the closest distance to the new data 
c) Calculate the weight voting from the data k. Classes with the highest voting value are labeled class for new data.

The weight value for each neighbor in the Modified K-Nearest Neighbor (MKNN) method is calculated based on Euclidean distance information and validity values. Weight calculation can be done using (10).

$$
W(x)=\operatorname{validity}(x) \times \frac{1}{\text { dist }_{\varepsilon}+0.5}
$$

Where $W(x)$ is weight voting of $\mathrm{x}$ data, validity $(\mathrm{x})$ is value of validity, dist $t_{\varepsilon}$ is euclidian distance.

\section{RESULTS AND ANALYSIS}

To find out the performance of the proposed method, two experiments scenario were carried out, manely the experiment to obtain the best features combination and the experiment to find the optimum value of K on MKNN. The feature combination test uses 180 traing data and the K value test uses 60,120 and 180 training data. Training data is used to get the best combination of features and the best number of $\mathrm{K}$ for classification with MKNN.

The best combination of features and $\mathrm{K}$ values obtained from the training process, are then used at the testing stage using some testing data. In each experiment, there are two treatments were conducted with different testing data, namely testing data 1 and testing data 2 . The number of data is 45 for testing data 1 and 95 for testing data 2 . Testing data 2 is a combination of testing data 1 and image pieces from different source images. The result of each experimet scenario is explained in the following section.

\subsection{Effects of feature combination on accuracy}

Feature combination testing aims to get the best feature combination to be used as input in the classification of soil images using the MKNN method. This test is performed by using 180 training data and the value of $\mathrm{K}$ is 3 . The results of combination feature tests are shown in Table 3, Table 4 and Table 5 .

Table 3 shows the result of experiment of the effect color feature combination. The using of color feature obtained the highest accuracy is $84.44 \%$ for the combination of mean and standard deviation features on data testing 1. Color Moments method itself specializes in feature extraction of images that have different levels of lighting. The experiment also shows that the less test data results in better accuracy. The less data has fewer variations in the value of pixels, therefore the classification results are more accurate.

Table 3. The effect of color feature combination on accuracy

\begin{tabular}{lcc}
\hline \multirow{2}{*}{ Feature } & \multicolumn{2}{c}{ Accuracy (\%) } \\
& Testing Data 1 & Testing Data 2 \\
\hline Mean & 73,33 & 60 \\
Deviation Standard & 26,67 & 31,58 \\
Mean + Deviation Standard & 84,44 & 69,47 \\
\hline
\end{tabular}

The next experiment is using the texture feature combination and the result is shown at Table 4. The result shows that the best accuracy is $80 \%$ on first testing data using a combination3, combination4, combination6, combination7, combination 9 and combination 12 . The same combination also gives the best result for the second testing data. Although this feature combination does not provide optimal results on third testing data, but the value of accuracy in this combination is quite stable. Therefore, these texture features combinations were then chosen by being combined with the best combination of color features. The result of combination test between color and texture features is shown at Table 5.

Table 5 shows the best accuracy is obtained when the combination of color features and texture features is applaid. There are four combinations of features that produce the best of accuracy at $93.33 \%$ for the testing data $1,63.16 \%$ for data testing 2 and $63.16 \%$ for data testing 3 . The first combination is a feature of the mean, standard deviation, contrast and entropy. The second combination is a feature of mean, standard deviation, contrast and IDM. The third combination is a feature of mean, standard deviation, contrast, ASM, and entropy. The last combination is the features of the mean, standard deviation, contrast, ASM, and IDM. These four combinations provide the most optimal results for all three tests data, while the remaining combinations provide unsatisfactory results. This shows that not all feature combinations will provide optimal results, but any combination that needs to be influential and has optimal results should be chosen. 
Table 4. The effect of GLCM features combination on accuracy

\begin{tabular}{lcc}
\hline \multirow{2}{*}{ Features } & \multicolumn{2}{c}{ Accuracy (\%) } \\
& Testing Data 1 & Testing Data 2 \\
\hline ASM & 33,33 & 15,79 \\
Contrass & 77,78 & 53,68 \\
IDM & 53,33 & 48,42 \\
Entropy & 40 & 23,16 \\
Correlation & 33,33 & 5,79 \\
Contrast+ASM & 77,78 & 53,68 \\
Contrast+Correlation & 53,33 & 48,42 \\
Contrast+Entropy & 80 & 52,63 \\
Contrast+IDM & 80 & 52,63 \\
Contrast+ASM+Correlation & 53,33 & 48,42 \\
Contrast+ASM+Entropy & 80 & 52,63 \\
Contrast+ASM+IDM & 80 & 52,63 \\
Contrast+Entropy+Correlation & 55,56 & 49,47 \\
Contrast+Entropy+IDM & 80 & 52,63 \\
Contrast+Correlation+IDM & 55,56 & 49,47 \\
Correlation+ASM+Contrast & 55,56 & 49,47 \\
Contrast+ASM+IDM+Entropy & 80 & 52,63 \\
Correlation+Contrast+ASM+Entropy & 33,33 & 15,79 \\
Correlation+Contrast+ASM+IDM & 55,56 & 49,47 \\
Correlation+Contrast+ASM+IDM+Entropy & 55,56 & 49,47 \\
\hline
\end{tabular}

Table 5. The effect of color and GLCM feature combination on accuracy

\begin{tabular}{lcc}
\multicolumn{1}{c}{ Feature } & \multicolumn{2}{c}{ Accuracy (\%) } \\
& Testing Data 1 & Testing Data 2 \\
\hline Mean+Deviation Standard+Contrast+Entropy & 93,33 & 63,16 \\
Mean+Deviation Standard+Contrast+IDM & 93,33 & 63,16 \\
Mean+Deviation Standard+Contrast+ASM+Entropy & 93,33 & 63,16 \\
Mean+Deviation Standard+Contrast+ASM+IDM & 93,33 & 63,16 \\
Mean+Deviation Standard+Contrast+Entropy+IDM & 35,56 & 27,37 \\
Mean+Deviation Standard+Contrast+ASM+IDM+Entropy & 33,33 & 31,58 \\
\hline
\end{tabular}

\subsection{Effects of variation in $K$ value against accuracy}

Experiment the $\mathrm{K}$ value on MKNN is done to find out the optimal number of neighbors for calculating the value of validity and class weights which are then used in the class determination process. The results of testing the combination of features obtained the best testing data is the Testing data 1 . Therefore, the testing of the effect of variations in the value of $\mathrm{K}$ used Testing data 1 , number of training data is 60,120 and 180, the $\mathrm{K}$ value tested is 1 to 15 . The result of this experiment is shown at Table 6 .

The results of testing the effect of variations in the $\mathrm{K}$ value with 60 training data found that the best accuracy is $77.78 \%$ when the $\mathrm{K}$ is $9,10,11$ and 12 . Whereas in the 120 training data obtained the best accuracy is $75.56 \%$ at the $\mathrm{K}$ is $1,2,9$, and 11 . The test results with 180 training data obtained the best accuracy is $93.33 \%$ when the $\mathrm{K}$ is 3 and 4 .

Based on the test results it can be seen that the addition of the amount of training data does not guarantee an improvement in the value of accuracy. This can be seen in the training data 120 does not have a higher accuracy when compared with testing with 60 training data. However, when the training data used is 180 data, the accuracy rate is better than using training data as much as 60 data and 120. This mean the addition of training data will improve the opportunity to get the closest neighbor that has the same class.

Table 6. The effect of K number on accuracy

\begin{tabular}{cccc}
\hline \multirow{2}{*}{ Number of K } & \multicolumn{3}{c}{ Accuracy $(\%)$} \\
& 60 Data & 120 Data & 180 Data \\
\hline 1 & 71,11 & 75,56 & 91,11 \\
2 & 71,11 & 75,56 & 91,11 \\
3 & 71,11 & 71,11 & 93,33 \\
4 & 73,33 & 73,33 & 93,33 \\
5 & 75,56 & 73,33 & 91,11 \\
6 & 75,56 & 73,33 & 91,11 \\
7 & 75,56 & 73,33 & 88,89 \\
8 & 75,56 & 73,33 & 91,11 \\
9 & 75,56 & 75,56 & 91,11 \\
10 & 77,78 & 73,33 & 91,11 \\
11 & 77,78 & 75,56 & 91,11 \\
12 & 77,78 & 71,11 & 88,89
\end{tabular}

olor moment and gray level co-occurrence matrix in classification of soil organic matter... (Candra Dewi) 


\begin{tabular}{cccc}
\hline \multirow{2}{*}{ Number of K } & \multicolumn{3}{c}{ Accuracy (\%) } \\
& 60 Data & 120 Data & 180 Data \\
\hline 13 & 71,11 & 73,33 & 88,89 \\
14 & 75,56 & 73,33 & 88,89 \\
15 & 71,11 & 73,33 & 91,11 \\
\hline
\end{tabular}

\section{CONCLUSION}

The Modified K-Nearest Neighbor (MKNN) algorithm is implemented to classify soil images based on the content of organic matter for essential planting. This method uses weight voting to the nearest neighbor as much as K, different from the usual K-Nearest Neighbor (KNN) method that uses a mode to determine the classification results. Based on the experiment that have been done, the MKNN method produces the best accuracy of $93.33 \%$ when using data testing 1, 63.16\% when using testing data 2 and $63.16 \%$ when using testing data 3 with a value of $\mathrm{K}=3$ on 180 training data. The best $\mathrm{K}$ value depends on the data used, where each dataset used has diferent of the best $\mathrm{K}$ value. The amount of training data also affects the level of accuracy, because the more of training data, the greater the chance of the nearest neighbor having the same class.

However, the more of features does not guarantee it will result the better of accuracy. The best results depent on the combination of the features used. The combination feature testing show that there are four best combination features for classification of soil images based on the content of organic material. The first combination is a feature of the mean, standard deviation, contrast and entropy. The second combination is a feature of mean, standard deviation, contrast and IDM. The third combination is a feature of mean, standard deviation, contrast, ASM, and entropy. The last combination is the features of the mean, standard deviation, contrast, ASM, and IDM. For the further reseach it is recommended to perform the automaticly feature selection. Therefore, the process of features selection can be done faster and more acurrate.

\section{ACKNOWLEDGEMENTS}

We would like to thank to Ministry of Research, Technology and Higher Education for the funding of this reseach.

\section{REFERENCES}

[1] M. Djazuli, "Effect of npk fertilizer and patchouli waste compost application on growth and productivity of patchouli," Buletin Littro, vol. 24, no. 2, pp. 87-92, 2013.

[2] S. P. More, M. A. Khan, S. P. R. Agrawal, "Soil nutrient detection through image processing in chromatogram image," International Journal of Pure and Applied Research in Engineering and Technology, vol. 2, no. 8, pp. 360-364, 2014.

[3] P.T. Sorenson, S.A. Quideau, B. Rivard, "High resolution measurement of soil organic carbon and total nitrogen with laboratory imaging spectroscopy," Geoderma, vol. 315, pp. 170-177, 2018.

[4] B. Wang, C. Waters, S. Orgill, J. Gray, A. Cowie, A. Clark, D. L. Liu, "High resolution mapping of soil organic carbon stocks using remote sensing variables in the semi-arid rangelands of eastern Australia," Science of The Total Environment, Vol. 630, pp. 367-378, 2018.

[5] A. Gholizadeh, D. Žižala, M. Saberioon, L. Borůvka, "Soil organic carbon and texture retrieving and mapping using proximal, airborne and Sentinel-2 spectral imaging," Remote Sensing of Environment, vol. 218, pp. 89-103, 2018.

[6] T. Wu, J. Luo, W. Dong, Y. Sun, L. Xia and X. Zhang, "Geo-object-based soil organic matter mapping using machine learning algorithms with multi-source geo-spatial data," IEEE Journal of Selected Topics in Applied Earth Observations and Remote Sensing, vol. 12, no. 4, pp. 1091-1106, 2019.

[7] X. Dou, X. Wang, H. Liu, X. Zhang, L. Meng, Y. Pan, Z. Yu, Y. Cui, "Prediction of soil organic matter using multi-temporal satellite images in the Songnen Plain, China," Geoderma, vol. 356, pp. 113896, 2019.

[8] K. J. Ward, S. Chabrillat, C. Neumann, S. Foerster, "A remote sensing adapted approach for soil organic carbon prediction based on the spectrally clustered LUCAS soil database," Geoderma, vol. 353, pp 297-307, 2019.

[9] N. Hamzehpour, H. Shafizadeh-Moghadam, R. Valavi, "Exploring the driving forces and digital mapping of soil organic carbon using remote sensing and soil texture," CATENA, vol. 182, pp. 104141, 2019.

[10] P. A. O. Morais, D. M. Souza, B. E. Madari, A. da S. Soares, A. E. Oliveira, "Using image analysis to estimate the soil organic carbon content," Microchemical Journal, vol. 147, pp 775-781, 2019.

[11] C. Dewi and Y. K. Arbawa, "Performance evaluation of distance function in KNN and WKNN for classification of soil organic matter," 2019 International Conference on Sustainable Information Engineering and Technology (SIET), Lombok, Indonesia, pp. 196-199, 2019.

[12] M. V. Nathan. Soils, "Plant nutrition and nutrient management," University of Missouri Extension. 2009. [Online]. Available: http://extension.missouri.edu/p/MG4. 
[13] F. Mindru, T. Tuytelaars, L. V. Gool, T. Moons, "Moment invariants for recognition under changing viewpoint and illumination," Computer Vision and Image Understanding, vol. 94, no. 1-3, pp. 3-27, 2004.

[14] H. Zhou, Y. Shen, Xinghui, Z. Bo, L. Zigang, FuNaFan, "Digital image modification detection using color information and its histograms," Forensic Science International, vol. 266, pp. 379-388, 2016.

[15] M. Gong, Y. Hao, H. Mo, H. Li, "Naturally combined shape-color moment invariants under affine transformations," Computer Vision and Image Understanding, vol. 162, pp. 46-56, 2017.

[16] L.K. Pavithra and T. S. Sharmila, "An efficient framework for image retrieval using color, texture and edge features," Computers and Electrical Engineering. vol. 70, pp. 580-593, 2018.

[17] C. Dewi, A. Basuki, "Identifying citronella plants from uav imagery using support vector machine," Telkomnika, vol. 16, no. 4, pp. 1877-1885, 2018.

[18] M.H. Abd Latif, H.Md. Yusof, S.N. Sidek, N. Rusli, "Implementation of GLCM features in thermal imaging for human affective state detection," Procedia Computer Science, vol. 76, pp. 308-315, 2015.

[19] C. Malegori, L. Franzetti, R. Guidetti, E. Casiraghi, R. Rossi, "GLCM, an image analysis technique for early detection of biofilm," Journal of Food Engineering, vol. 185, pp. 48-55, 2016.

[20] X. Ou, W. Pan, P. Xiao, "In vivo skin capacitive imaging analysis by using grey level co-occurrence matrix (GLCM)," International Journal of Pharmaceutics, vol. 460, no. 1-2, pp. 28-32, 2014.

[21] R. B. Vallabhaneni, V. Rajesh, "Brain tumour detection using mean shift clustering and GLCM features with edge adaptive total variation denoising technique," Alexandria Engineering Journal, vol. 57, no. 4, pp. 2387-2392, 2018.

[22] I. Saini, D. Singh, A. Khosla, "QRS detection using $K$-Nearest neighbor algorithm (KNN) and evaluation on standard ECG databases," Journal of Advanced Research, vol. 4, no. 4, pp. 331-344, 2013.

[23] X. Zhou, S.W. Gang, C. Li, D. Shou, "KNN processing with co-space distance in solomo systems," Expert Systems With Applications, vol. 41, no. 16, pp. 6967-6982, 2014.

[24] C. YunYang, J. Jen Chou, "A comparative evaluation approach for the classification of rotifers with modified nonparametric KNN," Image and Vision Computing, vol. 23, issue 4, pp. 427-439, 2005.

[25] F.R. Marcos, et al, "Effect of $\mathrm{ZnO}$ on the structure, microstructure and electrical properties of KNN-modified piezoceramics," Journal of the European Ceramic Society, vol. 29, no. 14, pp. 3045-3052, 2009.

[26] X. Vendrell, J.E. García, F. Rubio-Marcos, D.A. Ochoa, L. Mestres, J.F. Fernández, "Exploring different sintering atmospheres to reduce nonlinear response of modified KNN piezoceramics," Journal of the European Ceramic Society, vol. 33, no. 4, pp. 825-831, 2013.

[27] Z. Cen, Y. Zhen, W. Feng, P. Zhao L. Chen, X. Wang, L. Li, "Sintering temperature effect on microstructure, electrical properties and temperature stability of Mno-Modified KNN-Based ceramics," Journal of the European Ceramic Society, vol. 38, no. 9, pp. 3136-3146, 2018.

[28] Plantation Agency of East Java Province. 2013. Budidaya Tanaman Nilam. [Online]. Available : http://disbun.jatimprov.go.id.

[29] B.W. Murphy, Soil Organic Matter and Soil Functional - Review of the Literature and Underlying Data, Australia: Department of the Environment, 2014.

[30] W.L. Martinez and A.R. Martinez, Computational Statistics Handbook With Matlab, Florida : CRC Press LLC, 2002.

[31] H. Parvin, H. Alizadeh and B. Minati, "A modification on K-Nearest neighbor classification," Global Journal of Computer Science and Technology, vol. 10, no. 14, pp. 37-41, 2010. 